\title{
Erratum
}

\section{Erratum to "More Private Schools for Nonnative Students? Migrant Performance in Private Schools of Differing National Contexts"}

\author{
Monika Jungbauer-Gans ${ }^{1}$ and Christiane Gross ${ }^{2}$ \\ ${ }^{1}$ University of Erlangen-Nuremberg, Findelgasse 7/9, 90402 Nuremberg, Germany \\ ${ }^{2}$ Institute of Social Sciences, University of Kiel, Westring 400, 24098 Kiel, Germany \\ Correspondence should be addressed to Christiane Gross, cgross@soziologie.uni-kiel.de \\ Received 1 June 2011; Accepted 6 June 2011 \\ Copyright (C) 2011 M. Jungbauer-Gans and C. Gross. This is an open access article distributed under the Creative Commons \\ Attribution License, which permits unrestricted use, distribution, and reproduction in any medium, provided the original work is \\ properly cited.
}

This is the erratum paper for Tables 6 and 7, which were previously published in our article on pages 11 and 12 . Please note the correct placement of the effects marked in bold.

TABLE 6: Hierarchical multilevel models: determinants of mathematics competencies.

\begin{tabular}{|c|c|c|c|c|}
\hline \multirow{2}{*}{ Model } & (1) & (2) & (3) & (4) \\
\hline & Coeff. (T-ratio) & Coeff. (T-ratio) & Coeff. (T-ratio) & Coeff. (T-ratio) \\
\hline \multicolumn{5}{|c|}{ Fixed effects } \\
\hline Intercept & $464.67(56.83)^{* * *}$ & $501.22(14.84)^{* * *}$ & $441.95(12.22)^{* * *}$ & $261.14(7.48)^{* * *}$ \\
\hline \multicolumn{5}{|l|}{ Student level (level 1$)$} \\
\hline Migrant & $-19.32(-7.46)^{* * *}$ & $-19.37(-7.46)^{* * *}$ & $-18.57(-7.06)^{* * *}$ & $-13.49(-5.96)^{* * *}$ \\
\hline Female & & & & $-16.99(-70.32)^{* * *}$ \\
\hline HISEI & & & & $0.54(61.90)^{* * *}$ \\
\hline Age & & & & $8.92(21.65)^{* * *}$ \\
\hline \multicolumn{5}{|l|}{ No. of books at home ${ }^{(\mathrm{a})}$ : } \\
\hline $11-25$ & & & & $8.12(17.56)^{* * *}$ \\
\hline $26-100$ & & & & $24.18(63.83)^{* * *}$ \\
\hline $101-200$ & & & & $35.08(75.79)^{* * *}$ \\
\hline $201-500$ & & & & $49.95(98.96)^{* * *}$ \\
\hline$>500$ & & & & $48.96(77.83)^{* * *}$ \\
\hline \multicolumn{5}{|l|}{ School level (level 2) } \\
\hline \multicolumn{5}{|l|}{ School type $e^{(\mathrm{b})}$} \\
\hline Private school (indep.) & $39.91(6.90)^{* * *}$ & $18.22(0.59)$ & $11.91(0.49)$ & $9.73(0.42)$ \\
\hline Private school (gov. depend.) & $11.81(1.99)^{*}$ & $4.90(0.14)$ & $-30.86(-1.25)$ & $-28.42(-1.20)$ \\
\hline
\end{tabular}


TABle 6: Continued.

\begin{tabular}{|c|c|c|c|c|}
\hline Model & $\begin{array}{c}(1) \\
\text { Coeff. (T-ratio) }\end{array}$ & $\begin{array}{c}(2) \\
\text { Coeff. (T-ratio) }\end{array}$ & $\begin{array}{c}(3) \\
\text { Coeff. (T-ratio) }\end{array}$ & $\begin{array}{c}(4) \\
\text { Coeff. (T-ratio) }\end{array}$ \\
\hline Prop. girls & & & $11.65(5.05)^{* * *}$ & $23.17(10.71)^{* * *}$ \\
\hline Prop. migrants & & & $-25.72(-9.87)^{* * *}$ & $-23.05(-9.44)^{* * *}$ \\
\hline Prop. parents with tertiary edu. & & & $157.67(63.42)^{* * *}$ & $118.60(50.12)^{* * *}$ \\
\hline Prop. certified teachers & & & $2.29(1.47)$ & $2.18(1.50)$ \\
\hline School size & & & $0.01(15.56)^{* * *}$ & $0.01(15.23)^{* * *}$ \\
\hline Student-teacher ratio & & & $-0.08(-1.28)$ & $-0.08(-1.31)$ \\
\hline \multicolumn{5}{|l|}{ Community size ${ }^{(\mathrm{c})}$ : } \\
\hline $3,000-15,000$ & & & $3.02(2.02)^{*}$ & $1.92(1.38)$ \\
\hline $15,000-100,000$ & & & $3.24(2.18)^{*}$ & $1.47(1.06)$ \\
\hline $100,000-1,000,000$ & & & $0.43(0.27)$ & $-1.76(-1.19)$ \\
\hline$>1,000,000$ & & & $2.69(1.39)$ & $0.11(0.06)$ \\
\hline \multicolumn{5}{|l|}{ Country level (level 3) } \\
\hline Gini-Index (2005) & & $-1.72(-2.06)^{*}$ & $-1.74(-1.95)$ & $-1.49(-1.77)$ \\
\hline GNP per capita (2005) & & $0.00(2.83)^{* *}$ & $0.00(2.99)^{* *}$ & $0.00(2.96)^{* *}$ \\
\hline \multicolumn{5}{|l|}{ Cross-level effects } \\
\hline Gini* priv. school (indep.) & & $0.98(1.40)$ & $0.09(0.17)$ & $0.06(0.11)$ \\
\hline Gini* priv. school (gov. dep.) & & $0.57(0.73)$ & $0.98(1.76)$ & $0.84(1.57)$ \\
\hline GNP*priv. school (indep.) & & $-0.00(-1.52)$ & $-0.00(-2.18)^{*}$ & $-0.00(-2.21)^{*}$ \\
\hline GNP* priv. school (gov. dep.) & & $-0.00(-1.17)$ & $-0.00(-0.44)$ & $-0.00(-0.26)$ \\
\hline Priv. school (indep.)* migrant & $11.34(3.22)^{* *}$ & $11.20(3.18)^{* *}$ & $10.23(2.54)^{*}$ & $8.55(2.26)^{*}$ \\
\hline Priv. school (gov. dep.)* migrant & $8.79(2.52)^{*}$ & $7.42(2.29)^{*}$ & $6.77(2.01)^{*}$ & $4.44(1.40)$ \\
\hline \multicolumn{5}{|c|}{ Random effects } \\
\hline$\delta($ error variance level 1$)$ & 4915 & 4916 & 4918 & 4557 \\
\hline$\varepsilon($ error variance level 2) & 3008 & 3009 & 2000 & 1719 \\
\hline$\gamma($ error variance level 3$)$ & 3767 & 2527 & 2642 & 2288 \\
\hline $\begin{array}{l}\text { Deviance (no. of estimated } \\
\text { parameters) }\end{array}$ & $4,562,423(31)$ & $4,562,406(37)$ & $4,557,370(47)$ & $4,526,061(55)$ \\
\hline$N_{L 1}$ & 398,750 & 398,750 & 398,750 & 398,750 \\
\hline$N_{L 2}$ & 14,365 & 14,365 & 14,365 & 14,365 \\
\hline$N_{L 3}$ & 57 & 57 & 57 & 57 \\
\hline
\end{tabular}

(a) Ref.: $<11$.

(b) Ref.: public school.

(c) Ref.: $<3,000$.

${ }^{*} P<.05$; $^{* *} P<.01$; *** $P<.001$ (two-tailed tests).

TABLE 7: Hierarchical multilevel models: determinants of reading competencies.

\begin{tabular}{|c|c|c|c|c|}
\hline \multirow{2}{*}{ Model } & (1) & (2) & (3) & $(4)$ \\
\hline & Coeff. (T-ratio) & Coeff. (T-ratio) & Coeff. (T-ratio) & Coeff. (T-ratio) \\
\hline \multicolumn{5}{|c|}{ Fixed effects } \\
\hline Intercept & $455.43(58.64)^{* * *}$ & $476.87(13.87)^{* * *}$ & $380.27(10.49)^{* * *}$ & $196.22(5.62)^{* * *}$ \\
\hline \multicolumn{5}{|c|}{ Student level (level 1) } \\
\hline Migrant & $-27.78(-9.67)^{* * *}$ & $-27.76(-9.63)^{* * *}$ & $-26.77(-9.13)^{* * *}$ & $-20.48(-7.57)^{* * *}$ \\
\hline Female & & & & $29.26(86.69)^{* * *}$ \\
\hline HISEI & & & & $0.57(66.66)^{* * *}$ \\
\hline Age & & & & $8.68(18.30)^{* * *}$ \\
\hline \multicolumn{5}{|c|}{ No. books at home ${ }^{(\mathrm{a})}$ : } \\
\hline $11-25$ & & & & $11.23(21.90)^{* * *}$ \\
\hline $26-100$ & & & & $25.83(53.67)^{* * *}$ \\
\hline $101-200$ & & & & $37.33(67.90)^{* * *}$ \\
\hline
\end{tabular}


TABle 7: Continued.

\begin{tabular}{|c|c|c|c|c|}
\hline Model & $\begin{array}{c}(1) \\
\text { Coeff. (T-ratio) } \\
\end{array}$ & $\begin{array}{c}(2) \\
\text { Coeff. (T-ratio) }\end{array}$ & $\begin{array}{c}(3) \\
\text { Coeff. (T-ratio) } \\
\end{array}$ & $\begin{array}{c}(4) \\
\text { Coeff. (T-ratio) } \\
\end{array}$ \\
\hline $201-500$ & & & & $49.61(77.46)^{* * *}$ \\
\hline$>500$ & & & & $46.67(66.86)^{* * *}$ \\
\hline \multicolumn{5}{|l|}{ School level (level 2) } \\
\hline \multicolumn{5}{|l|}{ School type $e^{(\mathrm{b})}$} \\
\hline Private school (indep.) & $43.89(7.55)^{* * *}$ & $20.86(0.68)$ & $21.21(0.91)$ & $22.55(1.03)$ \\
\hline Private school (gov. depend.) & $12.00(1.91)$ & $-2.69(-0.08)$ & $-35.49(-1.43)$ & $-33.31(-1.48)$ \\
\hline Prop. girls & & & $68.92(26.74)^{* * *}$ & $37.33(15.63)^{* * *}$ \\
\hline Prop. migrants & & & $-32.02(-10.82)^{* * *}$ & $-29.12(-10.54)^{* * *}$ \\
\hline Prop. parents with tertiary edu. & & & $151.68(57.07)^{* * *}$ & $112.76(45.35)^{* * *}$ \\
\hline Prop. certified teachers & & & $3.65(2.15)^{*}$ & $3.90(2.48)^{*}$ \\
\hline School size & & & $0.01(15.39)^{* * *}$ & $0.01(14.84)^{* * *}$ \\
\hline Student-teacher ratio & & & $-0.02(-0.29)$ & $-0.03(-0.40)$ \\
\hline \multicolumn{5}{|l|}{ Community size ${ }^{(\mathrm{c})}:$} \\
\hline $3,000-15,000$ & & & $4.93(2.99)^{* *}$ & $3.45(2.25)^{*}$ \\
\hline $15,000-100,000$ & & & $8.14(4.91)^{* * *}$ & $5.79(3.75)^{* * *}$ \\
\hline $100,000-1,000,000$ & & & $6.07(3.47)^{* * *}$ & $3.47(2.13)^{*}$ \\
\hline$>1,000,000$ & & & $9.72(4.49)^{* * *}$ & $6.84(3.39)^{* * *}$ \\
\hline \multicolumn{5}{|l|}{ Country level (level 3) } \\
\hline Gini-Index (2005) & & $-1.42(-1.68)$ & $-1.31(-1.47)$ & $-0.96(-1.14)$ \\
\hline GNP per capita (2005) & & $0.00(3.29)^{* * *}$ & $0.00(3.48)^{* * *}$ & $0.00(3.34)^{* * *}$ \\
\hline \multicolumn{5}{|l|}{ Cross-level effects } \\
\hline Gini* priv. school (indep.) & & $1.06(1.53)$ & $0.11(0.21)$ & $-0.05(-0.09)$ \\
\hline Gini* priv. school (gov. dep.) & & $0.65(0.81)$ & $1.09(1.94)$ & $0.97(1.90)$ \\
\hline GNP* priv. school (indep.) & & $-0.00(-1.79)$ & $-0.00(-3.04)^{* *}$ & $-0.00(-3.10)^{* *}$ \\
\hline GNP* priv. school (gov. dep.) & & $-0.00(-0.66)$ & $-0.00(-0.16)$ & $0.00(0.08)$ \\
\hline Priv. school (indep.)* migrant & $5.98(1.44)$ & $5.99(1.42)$ & $5.82(1.34)$ & $3.69(0.92)$ \\
\hline Priv. school (gov. dep.)* migrant & $9.62(2.45)^{*}$ & $9.49(2.55)^{*}$ & $9.38(2.55)^{*}$ & $6.67(1.95)$ \\
\hline \multicolumn{5}{|c|}{ Random effects } \\
\hline$\delta$ (error variance level 1) & 5679 & 5678 & 5681 & 5211 \\
\hline$\varepsilon($ error variance level 2$)$ & 3645 & 3645 & 2426 & 2056 \\
\hline$\gamma($ error variance level 3$)$ & 3389 & 2386 & 2563 & 2248 \\
\hline $\begin{array}{l}\text { Deviance (no. of estimated } \\
\text { parameters) }\end{array}$ & $4,621,099(31)$ & $4,621,080(37)$ & $4,616,051(47)$ & $4,580,643(55)$ \\
\hline$N_{L 1}$ & 398,750 & 398,750 & 398,750 & 398,750 \\
\hline$N_{L 2}$ & 14,365 & 14,365 & 14,365 & 14,365 \\
\hline$N_{L 3}$ & 57 & 57 & 57 & 57 \\
\hline
\end{tabular}

(a) Ref.: $<11$.

(b) Ref.: public school.

(c) Ref.: $<3,000$.

${ }^{*} P<.05$ *** $^{*}<.01$; $^{* *} P<.001$ (two-tailed tests). 


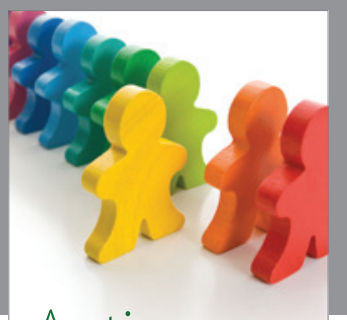

Autism

Research and Treatment
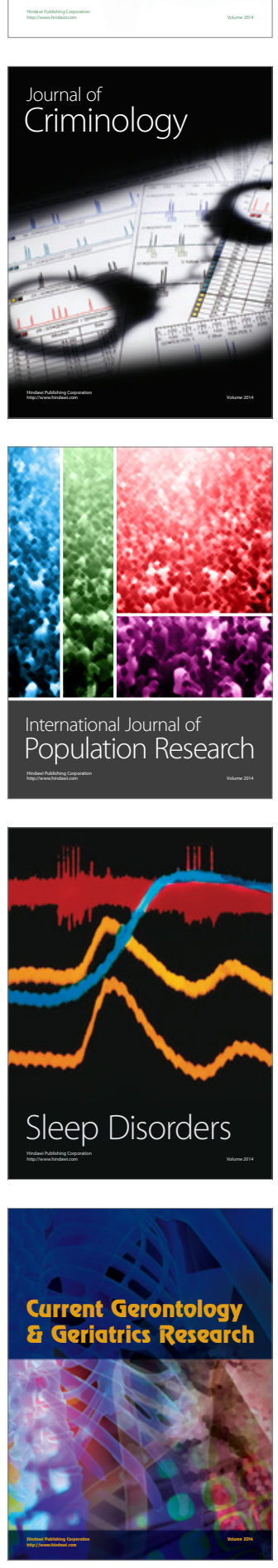
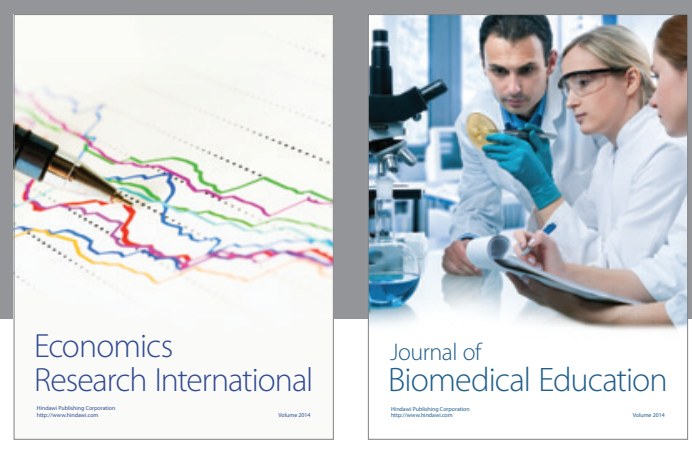

Journal of

Biomedical Education

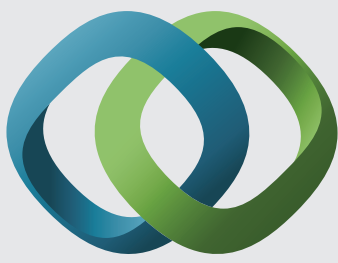

\section{Hindawi}

Submit your manuscripts at

http://www.hindawi.com
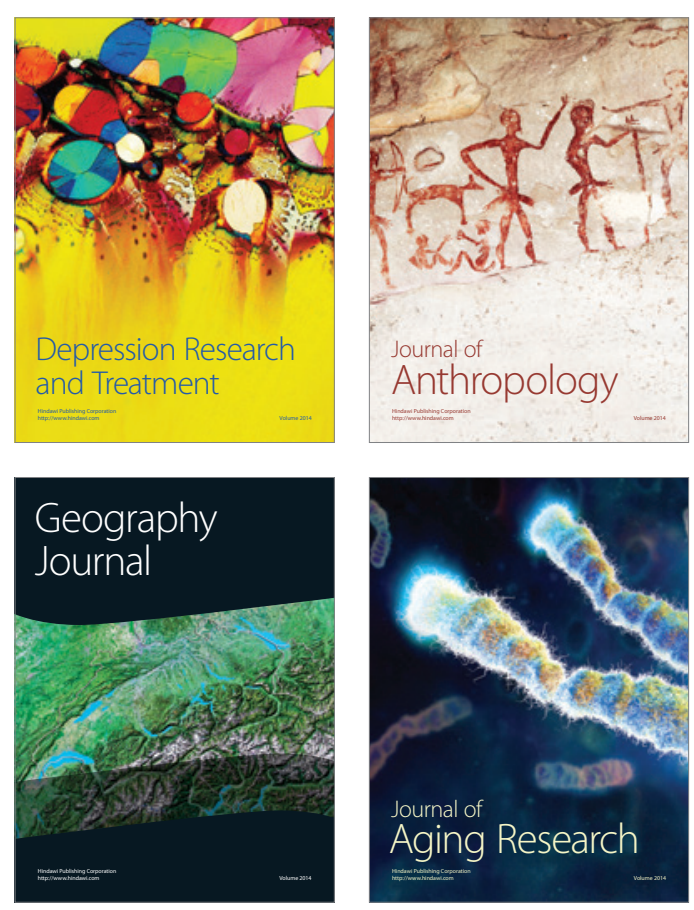

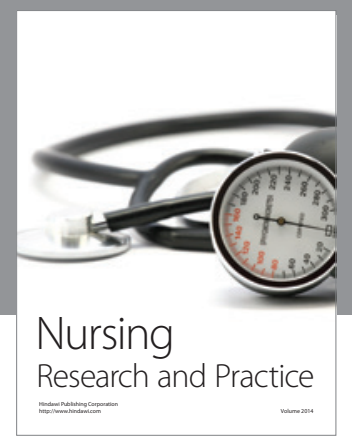

Nursing

Research and Practice

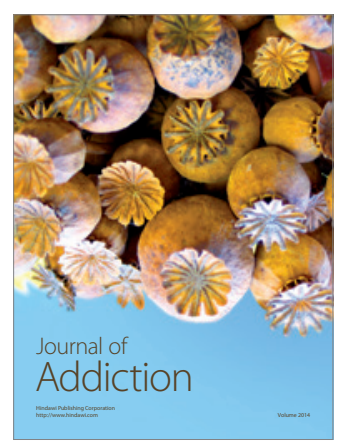

Child Development

Research

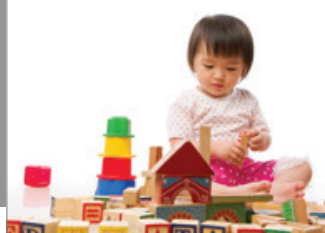

迥
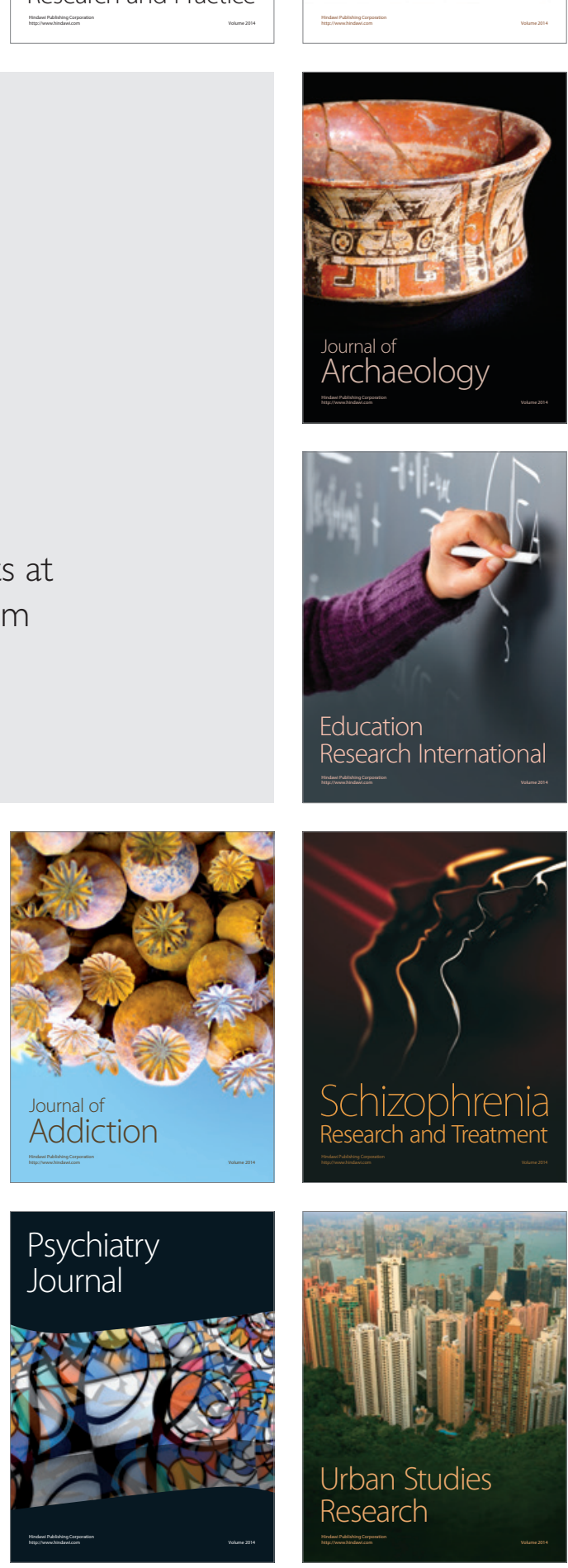